\title{
Differential Associations of Personal and General Just-World Beliefs with the Five- Factor and the HEXACO Models of Personality
}

Grégoire Bollmann $^{1,2}$, Franciska Krings ${ }^{1,3}$, Christian Maggiori ${ }^{1,4}$, and Jérôme Rossier ${ }^{1,2}$

${ }^{1}$ Swiss National Centre of Competence in Research LIVES, University of Lausanne, Switzerland; ${ }^{2}$ Institute of Psychology, University of Lausanne, Switzerland; ${ }^{3}$ Faculty of Business and Economics, University of Lausanne, Switzerland; ${ }^{4}$ School of Social Work Fribourg, University of Applied Sciences and Arts Western Switzerland.

\section{Author Note}

Correspondence concerning this article should be addressed to Grégoire Bollmann, National Centre of Competence in Research LIVEs, University of Lausanne, Géopolis Building, Office 5793, 1015 Lausanne, Switzerland. Email: gregoire.bollmann@ @nil.ch. Phone: +41216923857

Version: Uncorrected Proof

The final publication is available at Elsevier via http://dx.doi.org/j.paid.2015.08.020

(C) 2015, Bollmann. This manuscript version is made available under the CC-BY-NC-ND 4.0 license http://creativecommons.org/licenses/by-nc-nd/4.0/ 


\begin{abstract}
Recent literature evidences differential associations of personal and general just-world beliefs with constructs in the interpersonal domain. In line with this research, we examine the respective relationships of each just-world belief with the Five-Factor and the HEXACO models of personality in one representative sample of the working population of Switzerland and one sample of the general US population, respectively. One suppressor effect was observed in both samples: Neuroticism and emotionality was positively associated with general just-world belief, but only after controlling for personal just-world belief. In addition, agreeableness was positively and honesty-humility negatively associated with general justworld belief but unrelated to personal just-world belief. Conscientiousness was consistently unrelated to any of the just-world belief and extraversion and openness to experience revealed unstable coefficients across studies. We discuss these points in light of just-world theory and their implications for future research taking both dimensions into account.
\end{abstract}

Keywords: five-factor model; HEXACO model; just-world beliefs; personality models 


\section{Introduction}

People have a fundamental need to believe that the world is a just place where individuals get what they deserve and deserve what they get (Lerner, 1980). According to just-world theory, this need is essential because it helps people to harbor an illusion that the world is predictable, controllable and to derive meaning for their life. Everyone develops justworld beliefs, on the basis of a "personal contract" one makes with the world (Dalbert, 2001; Lerner, 1977). The terms of the contract stipulate that one would deserve and receive fairer outcomes and relationships by refraining from impulses and by making efforts in one's life. On this basis, people differ on the degree with which they believe the world treats them, personally, fairly; they have a personal just-world belief (i.e., personal JWB). Moreover, experiences of others are relevant to oneself to the extent that they provide relevant information for their own fate (Hafer, 2000). Accordingly, people also differ in the strength with which they believe that the world is just in general; they hold a general just-world belief (i.e, general JWB).

Personal and general JWB are positively interrelated. Despite this relation, they have some independent and even opposite effects. For instance, each belief has an opposite effect on the relation between mistreatment and victims' negative responses (Bollmann \& Krings, 2012; Strelan \& Sutton, 2011); while the personal JWB amplifies the relation between an unfair treatment and retribution, the general JWB attenuates it. Similarly, transgressors with a high general JWB rationalize their wrongdoing more than those with a low general JWB, whereas transgressors with a high personal JWB show the opposite tendency (Strelan \& Van Prooijen, 2014). Moreover, one just-world belief may suppress the other just-world belief's effect. For example, Sutton and Winnard (2007) found that general JWB increases young adults' delinquent intentions but only when the negative effect of personal JWB on these intentions is accounted for. According to these authors, an increased general JWB may foster 
the perception of blameworthiness of the (actual or potential) victim, decrease the guilty feelings of delinquents, and consequently facilitate antisocial attitudes. Until now however, opposite and suppressor effects have not been systematically explored. Furthermore, when examining relations between just-world beliefs and various dimensions of common personality models such as the five factor model, research has neither systematically investigated such effects, nor simultaneously considered personal and general just-world beliefs. Here, we seek to curb this gap with respect to the associations between people justworld beliefs and main dimensions of personality as considered by two widely used personality models.

A first objective of this research is to examine the relationship between personal JWB and the five-factor model of personality controlling for the general JWB, and inversely, between the general JWB and the five-factor model controlling for personal JWB. Given differences between the five-factor model and the HEXACO model of personality that considers among others an additional dimension, the second goal is to explore the same relations with the HEXACO model of personality instead of the five-factor model. In light of the differential effects each belief have in the interpersonal domain, unraveling their specific relations with known personality models is important because it might help better understand the reasons underlying these differential effects. It might also help generate new hypotheses about which type of outcomes is likely associated with which belief. In addition, delineating their respective relationships with various models of personality further contribute to establish the two beliefs as distinct but closely intertwined constructs, a point which is still often overlooked.

\subsection{Correlates of just-world beliefs}


Several studies assessed the relationship between different personal characteristics and JWB, using mostly Rubin and Peplau's (1975) scale, a proxy of general JWB. People strongly believing in the justice of the world have been shown to be more religious, more authoritarian, endorse a protestant work ethic, and to have a more trusting nature and a more internal locus of control (e.g., Rubin \& Peplau, 1975; Zuckerman \& Gerbasi, 1977; for reviews, Dalbert, 2009; Furnham \& Procter, 1989). In addition, people with high scores of general JWB endorse less self-directed values but more values related to conformism and security (Feather, 1991; Wolfradt \& Dalbert, 2003) and have more positive attitudes toward socio-political conservatism (for a review; Furnham, 2003).

More recent research has started to delineate the respective nomological network of personal and of general JWB. For example, interpersonal trust, religiosity and harsh social attitudes toward disadvantaged or minority people have been shown to be positively related to general but not to personal JWB (Bègue, 2002). Moreover, personal but not general JWB is related to more positive mood level, higher self-esteem, life satisfaction and, meaning in life (Bègue \& Bastounis, 2003; Dalbert, 1999; Sutton \& Douglas, 2005).

\subsection{JWB and FFM}

The five-factor model (FFM, McCrae \& Costa, 1999) is certainly the most accepted structure of personality traits. It suggests that people personality can be adequately described considering five dimensions, namely neuroticism, extraversion, openness, agreeableness, and conscientiousness. These main personality dimensions are known for being partly inheritable (Jang, McCrae, Angleitner, Riemann, \& Livesley, 1998), relatively stable over time (McCrae \& Costa, 1994) and across cultures (McCrae, Costa, Del Pilar, Rolland, \& Parker, 1998;

Zecca et al., 2013). 
Both personal and general JWB show similar but weak relations with the FFM dimensions. A recent meta-analysis including 12 studies and 2,579 participants indeed showed that both personal and general just-world beliefs are negatively related to neuroticism and positively related to extraversion and agreeableness (Nudelman, 2013). Nevertheless, coefficients were small in magnitude, with (absolute) values around or below .10, with the exception of the relation of personal JWB with neuroticism $(r=-.25)$ and extraversion $(r=.18)$. As Nudelman (2013) noted, some of these results need to be considered with caution. For some correlations (e.g., those including agreeableness), the limited number of studies prevents robust estimations.

More importantly for our purpose, previous studies neglected potential suppressing effects of the other just-world belief and those simultaneously examining the unique connections of each just-world belief with broad personality dimensions are scarce. For example, Lipkus and colleagues (1996) used several measures of personal and general JWB; but in their analyses, they did not examine personal and general JWB simultaneously. Wolfradt and Dalbert (2003) only focused on the general JWB when examining relations with the FFM. And Otto, Glaser and Dalbert (2009) included personal and general JWB in their study, but did not report results concerning the general JWB.

\subsection{JWB and HEXACO}

Besides the five-factor model, research on the HEXACO model has recently started to accumulate evidence for the existence of a sixth dimension of personality, honestyhumility. As the FFM, this model emerged from lexically-based studies of personality (Ashton \& Lee, 2007). It posits the existence of honesty-humility, composed of traits such as sincerity, fairness, greed avoidance and modesty, as an additional dimension complementing the more traditional five-factor model. Additionally, the contents of neuroticism (called 
emotionality, in the HEXACO framework) and agreeableness are slightly modified in this model. While the emotionality dimension of the HEXACO model includes the sentimentality related content of the agreeableness dimension of the FFM, its agreeableness dimension includes the anger related content of the neuroticism dimensions of the FFM. The remaining dimensions of extraversion, conscientiousness and openness to experience are closely related to their five-factor model counterparts.

To our knowledge, no study explored the relationships between the JWB and the HEXACO model of personality traits. Given that the content of two of the three dimensions of personality known to weakly correlate with just-world beliefs are modified in the HEXACO model, it is interesting to investigate whether the association between them and just-world beliefs remains similar. The dimension of honesty-humility might further deepen the understanding of personal and general just-world beliefs and their differences, for example, if it is differently related to them.

Moreover, honesty-humility and just-world beliefs have common correlates, which brings the question of the extent of their conceptual overlap. The honesty-humility dimension of HEXACO model indeed predicts outcomes such as the "Dark Triad"- - higher psychopathy, machiavellianism and narcissism—-materialism, delinquency or unethical decision making (Ashton \& Lee, 2008; Lee \& Ashton, 2005). Likewise, just-world beliefs play a role in delinquent behaviors and unethical decision-making (Ashkanasy, Windsor, \& Treviño, 2006; Begue \& Muller, 2006; Hafer, 2000; Sutton \& Winnard, 2007). And, they have been conceptually discussed in relation to psychopathy; individuals with strong general just-world beliefs and those with low scores of psychopathy report higher engagement in behaviors seen as deserving positive outcomes (Hafer, Bègue, Choma, \& Dempsey, 2005). Therefore, studying the link between just-world beliefs and honesty-humility would shed some light on whether their relation to these outcomes might be due to their overlap. 
Accordingly, Study 1 examined the unique associations of each just-world beliefs with personality dimensions measured with the FFM, using a large representative sample of working age people in Switzerland. Study 2 did the same, measuring personality dimensions with the HEXACO model and using a convenience sample of 138 participants from the general population in the United States.

\section{Study 1}

\subsection{Method}

\subsubsection{Participants}

Study 1 used data of an overall sample of 2,447 participants (50.7\% female; $\left.M_{\text {age }}=41.9, S D=8.63\right)$ who completed the first wave of a longitudinal research project hosted by [institution name]. More precisely, 1'987 participants stemmed from a random sample drawn by the Swiss Federal Statistics Office from the national register of inhabitants. This sample was representative of the Swiss working age population in terms of age, gender, and nationality. The other 460 participants represented a random sample provided by the State Secretariat for Economic Affairs, and were drawn from the national register of unemployed people.

\subsubsection{Procedure and materials}

The research protocol used in the first wave of the longitudinal project assessed individuals' professional situation and biography, their work and social environment, and their personal characteristics and resources. Participants could choose to answer on a paper-pencil or an online questionnaire in German or French and $82.9 \%$ of them opted for the online version while $62.2 \%$ completed the questionnaire in German (for a more detailed description 
of the protocol and method, see ANONYMIZED, 2013). Altogether, it took participants between 45 and 55 minutes to respond to the entire research protocol; at the end of the protocol they could choose a gift for an amount of 20 Swiss francs. Gift choices included a coupon for a supermarket, a department store, a library or a donation to a non-profit organization.

Personal and General JWB was measured with Dalbert's (1999) Personal Belief in a Just World Scale and General Belief in a Just World Scale. These are 6- and 7-item scales, respectively $(1=$ not true at all/strongly disagree; $6=$ very true/strongly agree $)$. Both scales were translated in German and French from the published version by native German- and French-speaking psychologists and then back translated by a native English-speaking psychologist. To ensure that the measurement of the two versions of the scale was invariant enough to perform unbiased comparisons, we conducted a multigroup confirmatory factor analysis between the German and French versions. For the fit indices, we considered that cutoff values above .95 for comparative fit index (CFI) and below .06 for root mean squared error of approximation (RMSEA) indicated good fit (Hu and Bentler, 1999), while values above .90 for CFI and below .08 are still acceptable (Byrne, 2001). Moreover, we relied on differences in fit indices to ensure that the next level of invariance was reached because $\chi^{2}$ difference tests are too sensitive to a large sample size (Cheung \& Rensvold, 2002). Existing standards suggest that a change of .01 in CFI and of .015 in RMSEA indicates a higher level of invariance (Chen, 2007). The model testing for configural invariance of the 13 items presented a good fit, $\chi^{2}\left(120, N=2^{\prime} 447\right)=577.77, p<.001, \mathrm{CFI}=.95, \mathrm{RMSEA}=.056(90 \%$ CI: .051, .060). With respect to metric invariance, the model also presented a good fit when freeing the equality constrain between loadings of one item in each dimension, $\chi^{2}(131, N=$ 2’447) $=646.46, p<.001, \mathrm{CFI}=.95, \mathrm{RMSEA}=.057$ (90\% CI: .052, .061). Neither CFI, nor RMSEA meaningfully changed, thus providing evidence for partial metric invariance. After 
freeing the intercept of an additional item, fit indices for the scalar invariance model were acceptable and changes in CFI and RMSEA did not exceed Chen's standards, $\chi^{2}(139, N=$ $\left.{ }^{\prime}{ }^{\prime} 447\right)=787.98, p<.001, \mathrm{CFI}=.94, \mathrm{RMSEA}=.062(90 \% \mathrm{CI} . .058, .066)$. Partial scalar invariance was thus reached; this implied that the French and German versions could be pooled without any bias resulting from the measurement. The mean difference in general JWB between German- and French-speaking respondents was not significant, $M_{\text {German }}=3.14$, $M_{\text {French }}=3.14, t(2445)=-0.24, p=.813$, Cohen's $d=-0.01$. However, the difference in personal JWB had a medium effect size, $M_{\text {German }}=4.34, M_{\text {French }}=3.94, t(2445)=11.13, p<$ .001 , Cohen's $d=0.46$.

The Five-factor model was measured using the French (Aluja, Garcia, Rossier, \& Garcia, 2005) and German (Schmitz, Hartkamp, Baldini, Rollnik, \& Tress, 2001) versions of the NEO Five Factor Inventory Revised (McCrae \& Costa Jr, 2004). Participants indicated their level of agreement with the 60 items on a 5-point scale $(1=$ strongly disagree, $5=$ strongly agree). Mean differences in the five personality dimensions were all significant $(p s<.05)$. Effect sizes were trivial for openness to experience, agreeableness and conscientiousness (all Cohen's $d<.11$ ) and small for neuroticism, Cohen's $d=-.24$, and extraversion, Cohen's $d=-.32$.

\subsection{Results}

\subsubsection{Correlations}

Mean, standard deviations, alpha reliabilities and correlations of Study 1 variables are displayed in Table 1. Pearson's coefficients (i.e., $r$ ) were computed for continuous variables and point-biserial correlations (i.e., $r_{\mathrm{pb}}$ ) for correlations involving gender. The correlation between personal JWB and general JWB was positive but small, $r=.23, p<.001$. 
Personal JWB negatively correlated with neuroticism, $r=-.35, p<.001$, while it presented positive and significant correlations with extraversion, openness to experience, and conscientiousness, ranging between .06 and .10. General JWB showed significant negative correlations with neuroticism, and openness to experience and positive ones with extraversion and conscientiousness, all of them ranging between -.09 and .10. In line with Nudelman's (2013) meta-analytic results, the correlation between personal JWB and neuroticism was the strongest observed; however contrary to his findings, neither personal JWB, $r=.02, p=.244$, nor general JWB, $r=.04, p=.069$, significantly correlated with agreeableness.

\subsubsection{Hierarchical regressions}

To isolate the part of the variance in the FFM which is unique to each dimension of JWB, two series of hierarchical regressions were computed, one with personal JWB and one with general JWB as the criterion. The five dimensions of the FFM were entered in a first step to produce regression coefficients which controlled for the correlations between them. In a second step, the other dimension of JWB was entered as a predictor, thus allowing observing unique association of each dimension of the FFM with the focal JWB dimension. Neither gender, nor age were included as control variables in the regressions results reported because preliminary tests showed they predicted a negligible part of the variance in personal JWB, $R^{2}=.002, F(2,2444)=2.77, p=.063$, and general JWB, $R^{2}=.00$, $F(2,2444)=1.13, p=.322$. Multicollinearity between the variables of interest (i.e., dimensions of the FFM and JWB) was not a problem since variance inflation factors in all regressions varied between 1.04 and 1.35. As a rule of thumb, values above 10 are considered diagnostic of the presence of multicollinearity (Belsey, Kuh, \& Welsch, 1980).

With respect to the first step of the regression with personal JWB as a criterion

(Table 2), neuroticism showed the strongest association, $\beta=-.37, p<.001$. Extraversion also 
revealed a significant negative, albeit small, coefficient, and openness to experience a small positive and significant coefficient. In addition, the coefficients for agreeableness and conscientiousness were null. Altogether, the FFM explained $13 \%$ of the variance in personal JWB, $F(5,2441)=72.76, p<.001$. In the second step, controlling for general JWB, this pattern of results remained stable, yielding slightly stronger coefficients for extraversion and openness to experience. Overall, after controlling for general JWB, neuroticism presented the strongest relation of all the dimensions.

With respect to the regression with general JWB as a criterion, the first step explained only $4 \%$ of the variance in general JWB, $F(5,2441)=18.21, p<.001$. Openness to experience revealed the strongest coefficient, $\beta=-.15, p<.001$, followed by extraversion which, in contrast to the regression predicting personal JWB, was positively related to general JWB, $\beta=.11, p<.001$. Moreover, agreeableness was slightly positively related to general JWB and conscientiousness unrelated to it. Finally, neuroticism showed only a marginal negative coefficient for general JWB, $\beta=-.04, p=.051$. Importantly however, once personal JWB was controlled for, this coefficient became positive and significant, $\beta=.05, p=.032$. The remaining coefficients were similar to the ones revealed in Step 1.

\subsection{Discussion}

Results of Study 1 evidenced that only little variance of just-world beliefs was explained by the FFM and that the correlation between the two beliefs was small, indicating that both JWB dimensions capture distinct individual differences that personality dimensions of the FFM model only weakly cover. Regression coefficients between the FFM and personal JWB were small with the exception of neuroticism which was moderately related to personal JWB. They were also relatively stable after controlling for general JWB. Regression coefficients between general JWB and the FFM were small, too. Importantly however, 
Table 1

Means, Standard Deviations, Correlations and Reliabilities of Study 1 Variables

\begin{tabular}{|c|c|c|c|c|c|c|c|c|c|c|c|}
\hline & $M$ & $S D$ & 1 & 2 & 3 & 4 & 5 & 6 & 7 & 8 & 9 \\
\hline 1. Gender ${ }^{\mathrm{a}}$ & 0.51 & 0.50 & & & & & & & & & \\
\hline 2. Age (in years) & 41.90 & 8.63 & -.03 & & & & & & & & \\
\hline 3. Personal JWB & 4.19 & 0.88 & -.02 & $.04 *$ & $(.92)$ & & & & & & \\
\hline 4. General JWB & 3.14 & 0.90 & -.03 & -.01 & $.23 * * *$ & $(.80)$ & & & & & \\
\hline 5. Neuroticism & 2.68 & 0.53 & $.13 * * *$ & $-.09 * * *$ & $-.35 * * *$ & $-.09 * * *$ & $(.82)$ & & & & \\
\hline 6. Extraversion & 3.41 & 0.48 & $.09 * * *$ & -.04 & $.09 * * *$ & $.10^{* * *}$ & $-.32 * * *$ & $(.74)$ & & & \\
\hline 7. Openness to experience & 3.45 & 0.50 & $.15 * * *$ & $.08 * * *$ & $.06 * *$ & $-.11 * * *$ & -.00 & $.27 * * *$ & $(.74)$ & & \\
\hline 8. Agreeableness & 3.39 & 0.32 & $.15^{* * *}$ & $.10 * * *$ & .02 & .04 & -.04 & $.12 * * *$ & $.17 * * *$ & $(.71)$ & \\
\hline 9. Conscientiousness & 3.94 & 0.47 & $.09 * * *$ & $.08 * * *$ & $.10 * * *$ & $.07 * * *$ & $-.31 * * *$ & $.32 * * *$ & $.07 * * *$ & $.13 * * *$ & $(.80)$ \\
\hline
\end{tabular}

Note: $* p<.05 ; * * p<.01 ; * * * p<.001$. JWB = Just-World Beliefs. Reliabilities appear in parentheses.

${ }^{\mathrm{a}} 0=$ male, $1=$ female. 
Table 2

Results of Hierarchical Regressions of FFM on Personal JWB and General JWB

\begin{tabular}{|c|c|c|c|c|}
\hline \multirow[b]{2}{*}{ Variables } & \multicolumn{2}{|c|}{ Personal JWB } & \multicolumn{2}{|c|}{ General JWB } \\
\hline & Step 1 & Step 2 & Step 1 & Step 2 \\
\hline Neuroticism & $-0.37 * * *$ & $-0.36 * * *$ & -0.04 & $0.05^{*}$ \\
\hline Extraversion & $-0.05^{*}$ & $-0.07 * * *$ & $0.11 * * *$ & $0.12 * * *$ \\
\hline Openness to experience & $0.07 * *$ & $0.10 * * *$ & $-0.15 * * *$ & $-0.17 * * *$ \\
\hline Agreeableness & 0.00 & 0.00 & $0.04 *$ & $0.04 *$ \\
\hline Conscientiousness & 0.00 & -0.01 & 0.03 & 0.03 \\
\hline General JWB & & $0.22 * * *$ & & \\
\hline Personal JWB & & & & $0.24 * * *$ \\
\hline$R^{2}$ & 0.13 & 0.18 & 0.04 & 0.09 \\
\hline$\Delta R^{2}$ & & 0.05 & & 0.05 \\
\hline$F$ & $72.76^{* * *}$ & $87.30 * * *$ & $18.21 * * *$ & $39.26 * * *$ \\
\hline$d f$ & 5,2441 & 6,2440 & 5,2441 & 6,2440 \\
\hline
\end{tabular}

Note. $* p<.05 ; * * p<.01 ; * * * p<.001$. Standardized regression coefficients $(\beta)$ are shown. 
neuroticism revealed a positive association with general JWB after controlling for personal JWB. This stands in contrast with the moderate negative coefficient that neuroticism revealed with personal JWB and indicates that personal JWB suppressed the positive association between neuroticism and general JWB. Further comparing the results for personal and general JWB, the relation of extraversion and openness to experience with each JWB dimension were opposite in sign. Moreover, while agreeableness was not related to personal JWB, it showed a small positive relation with general JWB. Conscientiousness was the sole trait consistently unrelated to personal and general JWB.

Overall, this study showed that, the five dimensions of personality composing the FFM model present a different pattern of associations with personal and general JWB when controlling for the other belief. Importantly, these observations were made with a representative sample $(N=2,477)$ which size is comparable to the overall sample of Nudelman's meta-analysis $(N=2,579)$. Considering this, it could be informative to replicate the results with a different personality model such as the HEXACO. This model of personality additionally allows to study relations between just-world beliefs and a sixth dimension of honesty-humility.

\section{Study 2}

\subsection{Method}

\subsubsection{Participants}

We recruited Study 2 participants among US users of Mechanical Turk with a 95\% approval rating of their activity at least. Our sample was composed of 149 participants living in the US who receiving $\$ 2.00$ each for survey completion. We excluded data from 11 participants because their geolocation data indicated they were outside US or because they 
shared the same ip address. The final sample hence consisted of 138 participants ( 83 females, 55 males, $\left.M_{\text {age }}=34.44, S D=11.96\right)$.

\subsubsection{Procedure and material}

The survey took 25 minutes $(S D=13.54)$ to complete in average. Participants first gave demographic information and then completed the individual differences in JWB and the personality inventory, which were separated by another scale irrelevant to this study. We inserted 3 items checking participants' attention, one among the JWB items and two among the personality items. These items instructed participants to choose a specific response option (e.g., For this specific item, please click "Strongly disagree (1)"). Whenever participants did not choose the correct response option, they received a warning message asking them to read all instructions and items before answering; they were forced to correct their answer before proceeding to the next page.

Personal and General JWB were assessed using the English-version of the Personal Belief in a Just World Scale and General Belief in a Just World Scale developed by Dalbert (1999).

The 100 items of the revised version of HEXACO personality inventory (Lee \& Ashton, 2004) were used to assessed personality traits of honesty-humility, emotionality, extraversion, agreeableness, conscientiousness and openness to experience. Participants indicated their level of agreement with statements describing them on a 5 point scale $(1=$ strongly disagree, 5 = strongly agree).

\subsection{Results}

\subsubsection{Correlations}


Table 3 present the means, standard deviation, reliabilities and correlations of Study 2 variables. The correlation between personal JWB and general JWB, $r=.60, p<.001$, was much stronger than in Study 1. Similar to Nudelman's (2013) meta-analytic results, both personal and general JWB had significant and positive correlations with extraversion and agreeableness while they did not reliably correlate with conscientiousness or openness to experience. In contrast to the meta-analysis however, neither personal JWB nor general JWB correlated with emotionality. Lastly, they revealed non-significant correlations with honestyhumility.

\subsubsection{Hierarchical regressions}

In the second part of the analyses, as in Study 1, we isolated the part of the variance in personality dimensions as measured by the HEXACO model which uniquely pertained to each dimension of JWB. First, we regressed each just-world belief on the six personality dimensions, and then added the other just-world belief to partial out its common variance with the criterion belief. Multicollinearity was not a concern among the predictors; variance inflation factors ranged between 1.03 and 1.30 .

As shown in Step 1 of Table 4, extraversion, $\beta=.35, p<.001$, and agreeableness, $\beta=$ $.20, p=.021$, were significantly related to personal JWB but neither honesty-humility, emotionality, conscientiousness nor openness to experience revealed significant coefficients. Overall, this regression explained $19 \%$ of the variance in personal JWB, $F(6,131)=5.06, p<.001$. In Step 2, controlling for general JWB, the coefficients for extraversion and agreeableness dropped, even though the first one remained significant. Importantly, in this step emotionality emerged as a significant predictor of personal JWB, $\beta=-.17, p=.016$. 
Table 3

Means, Standard Deviations, Correlations and Reliabilities of Study 2 Variables

\begin{tabular}{|c|c|c|c|c|c|c|c|c|c|c|c|c|}
\hline & $M$ & $S D$ & 1 & 2 & 3 & 4 & 5 & 6 & 7 & 8 & 9 & 10 \\
\hline 1. Gender ${ }^{a}$ & 0.61 & 0.49 & & & & & & & & & & \\
\hline 2. Age & 35.76 & 12.50 & -.10 & & & & & & & & & \\
\hline 3. Personal JWB & 4.13 & 1.00 & .03 & $-.17^{\dagger}$ & $(.92)$ & & & & & & & \\
\hline 4. General JWB & 3.54 & 0.96 & .00 & .02 & $.60 * * *$ & $(.83)$ & & & & & & \\
\hline 5. Honesty-Humility & 3.57 & 0.76 & .14 & $.34 * * *$ & -.03 & -.06 & $(.84)$ & & & & & \\
\hline 6. Emotionality & 3.21 & 0.66 & $.42 * * *$ & -.08 & .00 & .07 & $.24^{*}$ & $(.83)$ & & & & \\
\hline 7. Extraversion & 3.06 & 0.77 & -.04 & .04 & $.46 * * *$ & $.41 * * *$ & -.01 & .00 & $(.89)$ & & & \\
\hline 8. Agreeableness & 3.10 & 0.72 & -.13 & .11 & $.23 *$ & $.26^{*}$ & $.34 * * *$ & -.07 & $.22 *$ & $(.90)$ & & \\
\hline 9. Conscientiousness & 3.79 & 0.60 & -.03 & $.27 * *$ & .11 & $.16^{\dagger}$ & $.17^{\dagger}$ & -.15 & $.26^{* *}$ & $.17^{\dagger}$ & $(.88)$ & \\
\hline 10. Openness to experience & 3.67 & 0.70 & -.09 & -.12 & .00 & -.11 & -.11 & -.08 & .10 & .09 & .03 & $(.80)$ \\
\hline
\end{tabular}

Note: ${ }^{\dagger} p<.10 ; * p<.05 ; * * p<.01 ; * * * p<.001$. JWB = Just-World Belief. Reliabilities appear in parentheses.

a $0=$ male, $1=$ female. 
Table 4

Results of Hierarchical Regressions of HEXACO Dimensions on Personal JWB and General JWB

\begin{tabular}{|c|c|c|c|c|}
\hline \multirow[b]{2}{*}{ Variables } & \multicolumn{2}{|c|}{ Personal JWB } & \multicolumn{2}{|c|}{ General JWB } \\
\hline & Step 1 & Step 2 & Step 1 & Step 2 \\
\hline Honesty-Humility & -0.05 & 0.05 & $-0.17 *$ & $-0.15^{*}$ \\
\hline Emotionality & -0.12 & $-0.17 *$ & 0.09 & $0.16^{*}$ \\
\hline Extraversion & $0.35^{* * *}$ & $0.19 *$ & $0.29 * *$ & 0.10 \\
\hline Agreeableness & $0.20 *$ & 0.02 & $0.32 * * *$ & $0.21 * *$ \\
\hline Conscientiousness & -0.04 & -0.04 & 0.01 & 0.03 \\
\hline Openness to experience & -0.11 & -0.01 & $-0.18^{*}$ & $-0.12^{\dagger}$ \\
\hline General JWB & & $0.57 * * *$ & & \\
\hline Personal JWB & & & & $0.54 * * *$ \\
\hline$R^{2}$ & 0.19 & 0.44 & 0.23 & 0.46 \\
\hline$\Delta R^{2}$ & & 0.25 & & 0.23 \\
\hline$F$ & $5.06 * * *$ & $14.40 * * *$ & $6.36 * * *$ & $15.99 * * *$ \\
\hline$d f$ & 6,131 & 7,130 & 6,131 & 7,130 \\
\hline
\end{tabular}

Note. ${ }^{\dagger} p<.10 ; * p<.05 ; * * p<.01 ; * * * p<.001$. Standardized regression coefficients $(\beta)$ are shown. 
With respect to general JWB, the six dimensions of the HEXACO model together explained $23 \%$ of its variance, $F(6,131)=6.36, p<.001$. Consistent with results of Study 1 , extraversion, $\beta=.29, p=.001$, agreeableness, $\beta=.32, p<.001$, and openness to experience, $\beta$ $=-.18, p=.022$, showed significant coefficients in the regression without controlling for personal JWB. In addition, honesty-humility revealed a negative relation with general JWB, $\beta$ $=-.17, p=.049$. Neither the coefficient for emotionality, nor the one for conscientiousness was significant.

When controlling for personal JWB in Step 2, extraversion and openness to experience dropped to non-significance while honesty-humility and agreeableness remained significant. Moreover, the coefficient for honesty-humility barely changed and the coefficient for agreeableness revealed somewhat smaller in magnitude. Importantly, similar to Study 1 , emotionality emerged as a significant and positive predictor of general JWB, $\beta=.16, p=$ .023 .

To further explore the previously unknown negative relationship between honestyhumility and general JWB, we conducted two additional regressions entering honestyhumility facets as sole predictors, with and without personal JWB as a control variable. Without personal JWB, fairness exhibited a positive, $\beta=.39, p<.001$, and sincerity a negative, $\beta=-.25, p=.008$, relationship with general JWB. In contrast, the coefficients for greed avoidance, $\beta=-.14, p=.133$, and modesty, $\beta=-.18, p=.057$, did not reach significance. Controlling for personal JWB, the coefficients for fairness, $\beta=.28, p<.001$, and sincerity, $\beta=-.15, p=.047$, dropped in magnitude but remained significant. In addition, the coefficient for modesty turned significant, $\beta=-.17, p=.026$, while the coefficient for greed avoidance was still not significant, $\beta=-.07, p=.338$.

\subsection{Discussion}


The pattern of correlations results obtained in Study 2 was similar for both dimensions of JWB to results of the meta-analysis by Nudelman (2013), with the notable exception of emotionality.

When considering regressions results, Study 2 showed that nearly half of the variance in personal and general JWB was explained by the HEXACO model and the general, respectively, personal JWB. This stands in contrast to Study 1 in which the second step of our regressions explained less than a fifth of the variance in personal and general JWB.

Additionally contradicting Study 1, personal JWB was positively predicted by extraversion but not by openness to experience. However, as in Study 1, emotionality again revealed a significant and negative, albeit small, relation with personal JWB. It emerged only when general JWB was controlled for. Agreeableness and conscientiousness were consistently unrelated to personal JWB, as in Study 1. With respect to general JWB, the pattern of findings was mostly comparable to Study 1 . Emotionality and agreeableness were significant predictors of general JWB, yet stronger ones than in Study 1. Importantly, personal JWB again suppressed the effect of emotionality on general JWB. In addition, conscientiousness and openness to experience revealed similar coefficients to Study 1. Extraversion however was not significantly related to general JWB, which stands in contrast with Study 1. Finally honesty-humility had a significant negative relationship with general JWB but no relationship with personal JWB. When considered at the facet level, this negative coefficient can be seen as consistent with a priori expectations; we discuss this point further on in the next section.

\section{General discussion}

The present research was aimed at examining the unique association of each justworld belief with the FFM and HEXACO models of personality. Overall, regressions results revealed that personality dimensions, be it measured with the FFM in Study 1, or the 
HEXACO model in Study 2 maintain different patterns of association with personal and general JWB. In what follows, we first review results that are consistent across studies, and then outline particularities pointing toward directions for future research.

Across studies, some consistent relations (or lack thereof) were observed between personal and general just-world beliefs and several broad personality dimensions, namely neuroticism, agreeableness, and conscientiousness. Starting with neuroticism (i.e., emotionality for the HEXACO model), a striking result of the regressions was the negative and respectively, positive relation that this dimension presented with personal and general JWB. The positive relation with general JWB was previously undocumented and emerged only when personal JWB was controlled for. This suggests that the overlap between personal and general JWB suppresses this positive relation. These results are consistent with two compatible views on just-world beliefs. The resourceful view of just-world beliefs (i.e., the view advancing the metaphor of the personal contract; Dalbert, 2001; Lerner, 1977) suggests that the belief in the fairness of one's own treatment, which brings people the force to invest in their social relationships, relies at least partly on the belief in the justness of the world itself. People indeed would have few reasons to make efforts in their life to receive fair outcomes if they cannot expect the world to be fair in return. This implies that the presence of both beliefs constitutes the resourceful force of people personal contract. When people, more particularly those more in need, believe in societal fairness, they can also believe in the fairness of their own treatment and hence invest in long-term efforts (Laurin, Fitzsimons, \& Kay, 2011). In addition, our results show that without this resourceful force (i.e., the common part of personal and general JWB which is controlled for in the $2^{\text {nd }}$ step of our regressions), the part of general JWB which is unique is positively related to neuroticism (or emotionality). This relation corresponds with the view of general JWB as a delusion (e.g., Jost, 1995). According to this view, people need to develop the belief in a generally just world to go about 
managing threats of unpredictability and uncertainty (Lerner, 1980). Because randomness is threatening (Tullett, Kay, \& Inzlicht, 2014), the general belief in a just world is seen as a mechanism that people maintain to cognitively defend themselves and psychologically reduce the perception of threat. Importantly however, it does not always effectively eliminate the source of these threats (Jost \& Hunyady, 2003). Hence, because of a need to deal with unpredictability, people who are more likely to find themselves in stressful situations and to experience threats, namely those high in neuroticism or emotionality, simultaneously report higher score on this belief.

In addition to this suppressor effect, agreeableness also showed a consistently positive relation with general JWB across the two studies. This indicates the existence of relational considerations linked to this belief. Although this might appear at odds with known victim blaming and derogation phenomena of general JWB, this idea stands in line with recent theoretical and empirical arguments maintaining that victim blaming and belief systems preserving the status-quo might partly rest on how much people value interpersonal relationships (Jost, Ledgerwood, \& Hardin, 2008; van Prooijen \& van den Bos, 2009). This also resonates with views of agreeableness as indicating a tendency for friendly compliance (Digman, 1990; Nudelman, 2013) or self-controlled communal behavior (Yao \& Moskowitz, 2015) and with the known positive relation between general JWB and authoritarian submissiveness (Rubin \& Peplau, 1975). Likewise, the non-significant relation between agreeableness and personal JWB goes along with recent indications that, although people high in personal JWB are generally less likely to manifest harmful behaviors toward others, they behave toward others as a function of their treatment (Bollmann \& Krings, 2012; Strelan \& Sutton, 2011). Overall, it is noteworthy to mention that the stability of the effects of agreeableness and neuroticism (i.e., emotionality for HEXACO model) was observed despite 
the differences of Study 1 and 2, namely the model of personality used (i.e., FFM and HEXACO model), and the studies cultural context and language.

With respect to conscientiousness, regression results revealed that it has no relation neither with personal nor with general JWB. This consistent lack of association is in line with Nudelman's (2003) meta-analytical results but goes against some previous research reporting a positive relationship between conscientiousness and general JWB (e.g., Wolfradt \& Dalbert, 2003). This could suggest that just-world beliefs may have no direct link with conscientiousness and the few correlations observed between conscientiousness and justworld beliefs could probably be better explained by the links that some facets of conscientiousness entertain with other dimensions of the FFM or the HEXACO models related to just-world beliefs. For example, the self-discipline or dutifulness facets of conscientiousness are conceptually close to the self-controlled and compliant nature of people high in agreeableness discussed above. Indeed, conscientiousness and agreeableness are both positively associated with self-regulatory effortful processes (Jensen-Campbell et al., 2002). Likewise, the fairness facet of honesty-humility emphasizes compliance with social norms. Turning to the results that were particular to each study, honesty-humility revealed a significant negative relationship with general JWB but not with personal JWB. This was partly due to the negative, yet non-significant relation of general JWB with facets conceptually related to humility (i.e., greed avoidance, modesty). In addition, general JWB was positively related to the fairness facet of honesty-humility and negatively related to its sincerity facet. This negative relationship between honesty-humility and general JWB is consistent with the higher delinquent intentions of general just-world believers (Sutton \& Winnard, 2007). At the same time however, the compliant nature of the fairness facet of honesty-humility contrasts with this observation, a point that future research will have to clarify. 
Openness to experience had a negative relation with general JWB in both studies, albeit significant in Study 1 but not in Study 2. Even though a relative lack of power of Study 2 might partly explain these mixed findings, this inconsistency parallels existing literature. Indeed, on the one hand a significant negative correlation between openness to experience and general JWB had previously been uncovered (Wolfradt \& Dalbert, 2003). On the other hand however, meta-analytic results suggest this correlation is not substantial (Nudelman, 2013). Openness to experience and personal JWB additionally showed an inconsistent relation across studies. Similarly, extraversion revealed highly unstable associations with each just-world belief. Considering extraversion and openness to experience are supposed to be relatively similar across the FFM and the HEXACO model, at the present state these results call for restraint in interpretation and for future research.

\subsection{Future research and conclusion}

More research is needed to examine potential moderators of the unstable correlations and regression results that were observed. As our studies took place in two different countries, culture might modify the relations between just-world beliefs and common personality traits we observed. Some countries cultural dimensions indeed have been shown to relate to justworld beliefs (Furnham, 1993) and both dimensions of just-world beliefs have different importance for people from different cultures (Wu et al., 2011). In addition, considering the magnitude of the correlation between personal and general JWB showed an important variation across studies, future research should also look at the structure of just-world beliefs dimensions across various contexts. Culture and more generally the social-economic context might well be responsible for the strength of the relation between personal and general JWB. Considering the difference observed in the score of personal JWB between German- and French-speaking respondents of Study 1, this last point also concerns them, their cultural 
background and place of residence. In Study 2, the proportion of variance in just-world beliefs that was explained by HEXACO dimensions was more important than in Study 1 when FFM dimensions were used. Whether this result is due to modifications to the content of emotionality and agreeableness dimensions and the additional honesty-humility dimension of the HEXACO model remains to be clarified by future research. Here again, as both studies were realized in different countries with questionnaires in different languages, any conclusion about the explanatory power of the FFM and HEXACO based on them would be hasty.

Overall, the pattern of relationships that general and personal just-world beliefs revealed with common personality traits was different from correlational evidence of the meta-analysis by Nudelman (2013). This further warrants their simultaneous consideration beyond that of traditional models of personality. For example, recent work has started to look at the way individual differences directly affect people perceptions of the fairness of procedures and interpersonal treatment at work (Bianchi \& Brockner, 2012) and the role of time in justice related questions (Fortin, Cojuharenco, Patient, \& German, 2014). Future research could thus look at the impact of just-world beliefs on perceptions of organizational fairness and how they affect changes of fairness perceptions over time. By showing the many differential relationships that personal and general JWB maintain with a very broad range of broad personality traits, our work sets forth the groundwork for a deeper understanding of the distinctive function of each just-world belief in peoples' lives. 


\section{References}

Aluja, A., Garcia, O., Rossier, J., \& Garcia, L. F. (2005). Comparison of the NEO-FFI, the NEO-FFI-R and an alternative short version of the NEO-PI-R (NEO-60) in Swiss and Spanish samples. Personality and Individual Differences, 38, 591-604. doi: 1016/j.paid.2004.05.014

Ashkanasy, N. M., Windsor, C. A., \& Treviño, L. K. (2006). Bad apples in bad barrels revisited: Cognitive moral development, just world beliefs, rewards, and ethical decision-making. Business Ethics Quarterly, 16, 449-473. doi: 10.2307/3857792

Ashton, M. C., \& Lee, K. (2007). Empirical, theoretical, and practical advantages of the HEXACO model of personality structure. Personality and Social Psychology Review, 11, 150-166. doi: 10.1177/1088868306294907

Ashton, M. C., \& Lee, K. (2008). The prediction of Honesty-Humility-related criteria by the HEXACO and Five-Factor Models of personality. Journal of Research in Personality, $42,1216-1228$.

Bègue, L. (2002). Beliefs in justice and faith in people: Just world, religiosity and interpersonal trust. Personality and Individual Differences, 32, 375-382. doi: 10.1016/S0191-8869(00)00224-5

Bègue, L., \& Bastounis, M. (2003). Two spheres of belief in justice: Extensive support for the bidimensional model of belief in a just world. Journal of Personality, 71, 435-463. doi: 10.1111/1467-6494.7103007

Begue, L., \& Muller, D. (2006). Belief in a just world as moderator of hostile attributional bias. British Journal of Social Psychology, 45, 117-126. doi: $10.1348 / 014466605 \times 37314$

Belsey, D. A., Kuh, E., \& Welsch, R. E. (1980). Regression diagnostics: identifying influential data and sources of colinearity. New York, NY: John Wiley.

Bianchi, E. C., \& Brockner, J. (2012). In the eyes of the beholder? The role of dispositional trust in judgments of procedural and interactional fairness. Organizational Behavior and Human Decision Processes, 118, 46-59. doi: 10.1002/job.1958

Bollmann, G., \& Krings, F. (2012). What role do just-world beliefs play in harmful responses to injustice? Paper presented at the Paper presented at the Academy of Management Annual Meeting, Boston, USA, August 3-7.

Byrne, B. M. (2001). Structural equation modeling with AMOS: basic concepts, applications, and programming. Mahwah, NJ: Lawrence Erlbaum. 
Chen, F. F. (2007). Sensitivity of goodness of fit indexes to lack of measurement invariance. Structural Equation Modeling: A Multidisciplinary Journal, 14, 464-504. doi: $10.1080 / 10705510701301834$

Cheung, G. W., \& Rensvold, R. B. (2002). Evaluating goodness-of-fit indexes for testing measurement invariance. Structural Equation Modeling: A Multidisciplinary Journal, 9, 233-255. doi: 10.1207/S15328007SEM0902_5

Dalbert, C. (1999). The world is more just for me than generally: About the personal belief in a just world scale's validity. Social Justice Research, 12, 79-98. doi: 10.1023/a:1022091609047

Dalbert, C. (2001). The justice motive as a personal resource: Dealing with challenges and critical life events. New York, NY: Kluwer Academic / Plenum.

Dalbert, C. (2009). Belief in a just world. In M. R. Leary \& R. H. Hoyle (Eds.), Handbook of Individual Differences in Social Behavior (pp. 288-297). New York, NY: The Guilford Press.

Digman, J. M. (1990). Personality structure: Emergence of the five-factor model. Annual review of psychology, 41, 417-440. doi: 10.1146/annurev.ps.41.020190.002221

Feather, N. T. (1991). Human Values, Global Self-Esteem, and Belief in a Just World. Journal of Personality, 59, 83-107. doi: 10.1111/j.1467-6494.1991.tb00769.x

Fortin, M., Cojuharenco, I., Patient, D., \& German, H. (2014). It is time for justice: How time changes what we know about justice judgments and justice effects. Journal of Organizational Behavior, n/a-n/a. doi: 10.1002/job.1958

Furnham, A. (1993). Just World Beliefs in Twelve Societies. The Journal of Social Psychology, 133, 317-329. doi: 10.1080/00224545.1993.9712149

Furnham, A. (2003). Belief in a just world: research progress over the past decade. Personality and Individual Differences, 34, 795-817. doi: 10.1016/S01918869(02)00072-7

Furnham, A., \& Procter, E. (1989). Belief in a just world: Review and critique of the individual difference literature. British Journal of Social Psychology, 28, 365-384. doi: 10.1111/j.2044-8309.1989.tb00880.x

Hafer, C. L. (2000). Investment in long-term goals and commitment to just means drive the need to believe in a just world. Personality and Social Psychology Bulletin, 26, 10591073. doi: 10.1177/01461672002611004 
Hafer, C. L., Bègue, L., Choma, B. L., \& Dempsey, J. L. (2005). Belief in a just world and commitment to long-term deserved outcomes. Social Justice Research, 18, 429-444. doi: $10.1007 / \mathrm{s} 11211-005-8569-3$

Jang, K. L., McCrae, R. R., Angleitner, A., Riemann, R., \& Livesley, W. J. (1998). Heritability of facet-level traits in a cross-cultural twin sample: Support for a hierarchical model of personality. Journal of Personality and Social Psychology, 74, 1556-1565. doi: 10.1037/0022-3514.74.6.1556

Jensen-Campbell, L. A., Rosselli, M., Workman, K. A., Santisi, M., Rios, J. D., \& Bojan, D. (2002). Agreeableness, conscientiousness, and effortful control processes. Journal of Research in Personality, 36, 476-489. doi: 10.1016/S0092-6566(02)00004-1

Jost, J. T. (1995). Negative illusions: Conceptual clarification and psychological evidence concerning false consciousness. Political Psychology, 16, 397-424. doi: $10.2307 / 3791837$

Jost, J. T., \& Hunyady, O. (2003). The psychology of system justification and the palliative function of ideology. European Review of Social Psychology, 13, 111-153. doi: $10.1080 / 10463280240000046$

Jost, J. T., Ledgerwood, A., \& Hardin, C. D. (2008). Shared reality, system justification, and the relational basis of ideological beliefs. Social and Personality Psychology Compass, 2, 171-186. doi: 10.1111/j.1751-9004.2007.00056.x

Laurin, K., Fitzsimons, G. M., \& Kay, A. C. (2011). Social disadvantage and the selfregulatory function of justice beliefs. Journal of Personality and Social Psychology, 100, 149-171. doi: 10.1037/a0021343

Lee, K., \& Ashton, M. C. (2004). Psychometric Properties of the HEXACO Personality Inventory. Multivariate Behavioral Research, 39, 329-358. doi: 10.1207/s15327906mbr3902_8

Lee, K., \& Ashton, M. C. (2005). Psychopathy, machiavellianism, and narcissism in the FiveFactor Model and the HEXACO model of personality structure. Personality and Individual Differences, 38, 1571-1582. doi: 10.1016/j.paid.2004.09.016

Lerner, M. J. (1977). The justice motive: Some hypotheses as to its origins and forms. Journal of Personality, 45, 1-52. doi: 10.1111/j.1467-6494.1977.tb00591.x

Lerner, M. J. (1980). The belief in a just world: A fundamental delusion. New York: Plenum Press.

Lipkus, I. M., Dalbert, C., \& Siegler, I. C. (1996). The importance of distinguishing the belief in a just world for self versus for others: Implications for psychological well-being. 
Personality and Social Psychology Bulletin, 22, 666-677. doi:

$10.1177 / 0146167296227002$

McCrae, R. R., \& Costa Jr, P. T. (2004). A contemplated revision of the NEO Five-Factor Inventory. Personality and Individual Differences, 36, 587-596. doi: 10.1016/S01918869(03)00118-1

McCrae, R. R., Costa, P. T., Del Pilar, G. H., Rolland, J.-P., \& Parker, W. D. (1998). Crosscultural assessment of the Five-Factor Model: The revised NEO personality inventory. Journal of Cross-Cultural Psychology, 29, 171-188. doi: 10.1177/0022022198291009

McCrae, R. R., \& Costa, P. T. J. (1994). The stability of personality: Observations and evaluations. Current Directions in Psychological Science, 3, 173-175. doi: $10.2307 / 20182303$

McCrae, R. R., \& Costa, P. T. J. (1999). A five-factor theory of personality Handbook of Personality: Theory and Research (Vol. 2, pp. 139-153).

Nudelman, G. (2013). The belief in a just world and personality: A meta-analysis. Social Justice Research, 26, 105-119. doi: 10.1016/S0191-8869(03)00040-0

Otto, K., Glaser, D., \& Dalbert, C. (2009). Mental health, occupational trust, and quality of working life: Does belief in a just world matter? Journal of Applied Social Psychology, 39, 1288-1315. doi: 10.1111/j.1559-1816.2009.00482.x

Rubin, Z., \& Peplau, L. A. (1975). Who believes in a just world? Journal of Social Issues, 31, 65-89. doi: 10.1111/j.1540-4560.1975.tb00997.x

Schmitz, N., Hartkamp, N., Baldini, C., Rollnik, J., \& Tress, W. (2001). Psychometric properties of the German version of the NEO-FFI in psychosomatic outpatients. Personality and Individual Differences, 31, 713-722. doi: 10.1016/S01918869(00)00173-2

Strelan, P., \& Sutton, R. M. (2011). When just-world beliefs promote and when they inhibit forgiveness. Personality and Individual Differences, 50, 163-168. doi: 10.1016/j.paid.2010.09.019

Strelan, P., \& Van Prooijen, J.-W. (2014). Rationalizers or realists? The effects of transgressors' just world beliefs within committed relationships. Personality and Individual Differences, 63, 41-46. doi: 10.1016/j.paid.2014.01.046

Sutton, R. M., \& Douglas, K. M. (2005). Justice for all, or just for me? More evidence of the importance of the self-other distinction in just-world beliefs. Personality and Individual Differences, 39, 637-645. doi: 10.1016/j.paid.2005.02.010 
Sutton, R. M., \& Winnard, E. J. (2007). Looking ahead through lenses of justice: The relevance of just-world beliefs to intentions and confidence in the future. British Journal of Social Psychology, 46, 649-666. doi: 10.1348/014466606x166220

Tullett, A. M., Kay, A. C., \& Inzlicht, M. (2014). Randomness increases self-reported anxiety and neurophysiological correlates of performance monitoring. Social Cognitive and Affective Neuroscience. doi: 10.1093/scan/nsu097

van Prooijen, J. W., \& van den Bos, K. (2009). We blame innocent victims more than I do: Self-construal level moderates responses to just-world threats. Personality and Social Psychology Bulletin, 35, 1528-1539. doi: 10.1177/0146167209344728

Wolfradt, U., \& Dalbert, C. (2003). Personality, values and belief in a just world. Personality and Individual Differences, 35, 1911-1918. doi: 10.1016/S0191-8869(03)00040-0

Wu, M. S., Yan, X., Zhou, C., Chen, Y., Li, J., Zhu, Z., . . Han, B. (2011). General belief in a just world and resilience: Evidence from a collectivistic culture. European Journal of Personality, 25, 431-442. doi: 10.1002/per.807

Yao, Q., \& Moskowitz, D. S. (2015). Trait agreeableness and social status moderate behavioral responsiveness to communal behavior. Journal of Personality, 83, 191-201. doi: 10.1111/jopy.12094

Zecca, G., Verardi, S., Antonietti, J.-P., Dahourou, D., Adjahouisso, M., Ah-Kion, J., . . Rossier, J. (2013). African Cultures and the Five-Factor Model of Personality: Evidence for a Specific Pan-African Structure and Profile? Journal of Cross-Cultural Psychology, 44, 684-700. doi: 10.1177/0022022112468943

Zuckerman, M., \& Gerbasi, K. C. (1977). Belief in a just world and trust. Journal of Research in Personality, 11, 306-317. doi: 10.1016/0092-6566(77)90039-3 